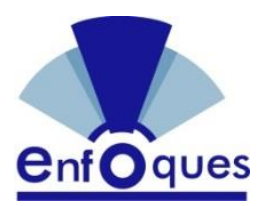

Aspectos importantes para la creación de una empresa de producción y comercialización de uva de mesa en la comunidad de Tárcana en Bolivia http://doi.org/10.33996/revistaenfoques.v1i3.15

No. 3 | Volumen 1 | Julio - Septiembre 2017 http://revistaenfoques.org ISSN: $2616-8219$

\section{ASPECTOS IMPORTANTES PARA LA CREACIÓN DE UNA EMPRESA DE PRODUCCIÓN Y COMERCIALIZACIÓN DE UVA DE MESA EN LA COMUNIDAD DE TÁRCANA EN BOLIVIA}

\author{
IMPORTANT ASPECTS FOR THE CREATION OF A TABLE GRAPE \\ PRODUCTION AND MARKETING COMPANY IN THE COMMUNITY OF \\ TÁRCANA IN BOLIVIA
}

Freddy Aramayo Escalante

\section{Resumen}

A partir de la investigación Proyecto de inversión para la creación de una empresa de producción y comercialización de uva de mesa en la comunidad de Tárcana, provincia Sud Cinti del departamento de Chuquisaca, realizada por el articulista, se plantea el presente estudio cuyo objetivo es poner en marcha la elaboración de un proyecto de inversión para lograr 10 hectáreas de uva de mesa de las variedades Flame y Sugraone en la comunidad de Tárcana. El estudio de mercado ayudó a determinar la cantidad que sería conveniente producir para hacer frente a los precios de la competencia, el diagnóstico que permite analizar los factores físicos de producción del lugar donde se quiere producir y el análisis financiero correspondiente. Según los resultados obtenidos, se puede concluir que es factible la creación de una empresa de producción de uva de mesa en la comunidad de Tárcana, para que sirva como referencia a los interesados en el tema.

Palabras clave: Producción; proyecto; investigación; inversión; Tárcana; actividad

\begin{abstract}
From the investigation Investment project for the creation of a table grape production and commercialization company in the community of Tárcana, Sud Cinti province of the department of Chuquisaca, carried out by the articulist, the present study whose objective is. To put into operation the development of an investment project to achieve 10 hectares of table grapes of the Flame and Sugraone varieties in the community of Tárcana. The market study helped to determine the quantity that would be convenient to produce to meet the prices of the competition, the diagnosis that allows to analyze the physical factors of production of the place where it is wanted to produce and the corresponding financial analysis. According to the results obtained, it can be concluded that the creation of a table grape production company in the community of Tárcana is feasible, to serve as a reference to those interested in the subject.
\end{abstract}

Key words: Production; project; research; investment; Tárcana; activity

Artículo recibido abril 2017 | Arbitrado entre mayo-junio 2017 | Publicado en julio 2017
Freddy Aramayo Escalante

fae_esca@hotmail.com

Universidad Adventista de Bolivia, Bolivia

Licenciado en Administración de Empresas de la Universidad Adventista de Bolivia. Consultor Independiente. Asistente Técnico contable, PROGRAMA EMPODERAR PARII Chuquisaca-Bolivia. Asistente Técnico en la Implementación de Sistemas de Riego. PROGRAMA ACCESOS Chuquisaca - Bolivia. 


\section{INTRODUCCIÓN}

Antes de entrar en el tema específico de este artículo, es importante señalar que un artículo de reflexión se entiende como un tipo de texto que "presenta resultados de investigación terminada desde una perspectiva analítica, interpretativa o crítica del autor, sobre un tema específico, recurriendo a fuentes originales" (Colciencias, 2010). Con esto se entiende que el artículo de reflexión es de carácter subjetivo en tanto permite el planteamiento de reflexiones, puntos de vista y valoraciones del investigador-autor, pero derivadas o sustentadas a partir de hallazgos de investigación.

Con relación a la temática de estudio, se quiere iniciar señalando que la uva es una fruta obtenida de la vid. Las uvas vienen en racimos, son pequeñas y dulces. Se comen frescas o se utilizan para producir agraz, mosto, vino y vinagre. Crecen grupadas en racimos de entre 6 y 300 uvas. Pueden ser negras, moradas, amarillas, doradas, púrpura, rosadas, marrones, anaranjadas o blancas, aunque estas últimas son realmente verdes y evolutivamente proceden de las uvas rojas con la mutación de dos genes que hace que no desarrollen antocianas, siendo estos los que dan la pigmentación. Como fruta seca se la llama pasa.

La mayoría de la uva cultivada proviene de la especie Vitis vinífera, natural de la Europa mediterránea y Asia central. En menor cantidad se producen en América y Asia. Según la Organización para la Alimentación y la Agricultura (FAO), la producción mundial de uva ocupa 75.866 kilómetros cuadrados. Aproximadamente el $71 \%$ de la producción es usada para vino, $27 \%$ consumo fresco y $2 \%$ como frutos secos. Una parte de la producción de zumo de uva es usada como edulcorante para zumos distribuidos bajo los lemas "sin azúcar añadido" y "100\% natural". El terreno dedicado a las viñas crece anualmente alrededor de $2 \%$. La uva se cosecha preferentemente a finales de verano, principios de otoño, en los climas mediterráneos.

Actualmente, la superficie cultivada en Bolivia es de 2.490 hectáreas, de las cuales $80 \%$ se encuentran en el valle de Tarija. Sin embargo, se podría decir que la producción en Bolivia es joven, pues esta cantidad es pequeña comparada a las 150 mil hectáreas cultivadas en Chile y a las 200 mil hectáreas en Argentina.

Para la producción de uva se requiere hacer uso de diferentes tecnologías como los sistemas de conducción, los sistemas de riego por goteo y de mallas antigranizo, además de actividades como la fertilización, las podas, fumigaciones, el cultivado, a fin de conseguir productos de calidad.

Durante las últimas décadas en la localidad de Tárcana, donde se efectuó la investigación referida, la producción de uva se ha visto afectada por la falta de financiamiento, factores climáticos que han ocasionado que la producción bajara de manera paulatina; también durante los últimos años las nuevas enfermedades y plagas han ocasionado que muchas plantaciones de vid se vean afectadas y fueran arrasadas.

La producción actual de uva en Bolivia no es suficiente para satisfacer la demanda que tiene el país en sus diferentes ciudades, el abastecimiento de este producto en los diferentes mercados del país es escaso y si bien existe el producto, estos son importados y tienen precios elevados de comercialización, por lo que muchas personas se ven limitadas a poder adquirirlo. Sin embargo, existen muchas tierras en 
diferentes lugares que no están siendo aprovechadas correctamente y que cumplen las condiciones para la producción de uvas y así poder ofertar este producto a los mercados del país.

La localidad de Tárcana cuenta con varias extensiones de terreno con la capacidad y las condiciones de producir uva las cuales no están siendo cultivadas adecuadamente, por lo que carece de los instrumentos y métodos adecuados de cultivo que mejoren la producción y el rendimiento de producción de los cultivos. EI proyecto de inversión pretende dar solución a estos dos problemas, respectivamente.

La falta de inversión e innovación en el sector ha provocado que la producción de uvas deje de ser una actividad económica importante. La falta de atención del Gobierno y de los productores ante los desastres naturales ha ocasionado que se pierdan cuantiosas plantaciones de viñedos, lo que ha ocasionado que los productores pierdan su inversión. En consecuencia, muchos productores han migrado a otros lugares del interior y exterior del país. EI resultado de la poca capacitación existente y la innovación en la producción de uvas ha incidido en los bajos índices de productividad y calidad.

Ante este panorama se formuló la siguiente interrogante: ¿es factible la creación de una empresa de producción y comercialización de uva de mesa mediante un proyecto de inversión en la comunidad de Tárcana, provincia Sud Cinti del departamento de Chuquisaca, en la gestión? Se asumió como hipótesis que un proyecto de inversión permitirá la creación de una empresa de producción y comercialización de uva de mesa en la comunidad antes mencionada.
Para ello se formuló como objetivo general diseñar un proyecto de inversión para la creación de una empresa de producción y comercialización de uva de mesa en la comunidad estudiada. Como objetivos específicos, analizar los factores que intervienen en la producción de uvas en la comunidad de Tárcana; elaborar un estudio de mercado para determinar la demanda y la oferta actual del mercado de la ciudad de Santa Cruz de la Sierra; elaborar un estudio técnico para la producción y comercialización de uvas en la comunidad de Tárcana; diseñar una estructura administrativo-organizacional para la empresa que responda de forma adecuada y permita desarrollar tanto las actividades como el quehacer de la producción y comercialización de uvas; realizar un análisis económico-financiero que permita viabilizar el proyecto de inversión y determinar su factibilidad.

\section{MATERIALES Y MÉTODO}

La modalidad de la investigación desarrollada en este artículo de reflexión fue de tipo cuantitativa. Midió entre ciertas alternativas usando magnitudes numéricas que pueden ser tratadas mediante herramientas del campo de la estadística. El tipo de investigación fue descriptivoexplicativo, lo que permitió ordenar el resultado de las observaciones de las conductas, las características, los factores, los procedimientos y otras variables de fenómenos y hechos. Mediante este método se buscó especificar propiedades, características y rasgos importantes de los fenómenos que fueron analizados.

Según Castillo (2004), el procedimiento empírico es el más antiguo por excelencia, pues consiste básicamente en utilizar los sentidos para observar los hechos, 
realidades sociales y las personas en su contexto cotidiano. Para que dicha observación tenga validez, es necesario que sea intencionada e ilustrada con un objetivo determinado y guía por un cuerpo de conocimiento. Se utilizó este método porque permitió observar el comportamiento, los hechos y realidades sociales de la comunidad de Tárcana con respecto a su actividad económica, las fuentes de trabajo de los habitantes y la necesidad de implementar una empresa de producción de viñedos que tenga la capacidad de crear nuevas fuentes de trabajo, además de rendir utilidades, y también contribuir al desarrollo $y$ crecimiento de la localidad de Tárcana.

La fuente y técnica de recolección de datos fue la entrevista, instrumento utilizado para obtener información de las fuentes. Se formularon 12 preguntas elaboradas de forma personal con el propósito de encontrar experiencias de otros productores y cuál es el concepto actual de la producción de uva. Asimismo, para conocer la opinión pública también se usó la encuesta y como herramienta los cuestionarios y su aplicación.

Con relación a la población y muestra del estudio, es necesario señalar que se consideró a 50 intermediarios de uvas en la ciudad de Santa Cruz de la Sierra. La muestra fue la totalidad de la población: 50 intermediarios.

Las encuestas a los comercializadores mayoristas tuvieron como objetivo conocer la demanda que tiene la ciudad de Santa Cruz y proponer mejores alternativas de adquisición de productos a los comerciantes. La entrevista tuvo como objetivo conocer la oferta existente, además de proponer un sistema adecuado de producción para tener rendimientos que generen utilidad a bajos costos para competir en el mercado.

Cuadro 1. Resumen del análisis de datos

\begin{tabular}{|c|c|c|c|c|}
\hline $\mathrm{N}^{\circ}$ & PREGUNTA & \multicolumn{3}{|c|}{ RESPUESTAS } \\
\hline \multirow{2}{*}{1} & \multirow{2}{*}{$\begin{array}{l}\text { ¿Dónde adquiere o compra la } \\
\text { uva? }\end{array}$} & PRODUCTOR & MERCADO & LUGAR DE VENTA \\
\hline & & 18 & 20 & 12 \\
\hline \multirow{2}{*}{2} & \multirow{2}{*}{$\begin{array}{l}\text { ¿Qué variedad de uva usted } \\
\text { comercializa? }\end{array}$} & FLAME & SUPERIOR & OTRAS \\
\hline & & 20 & 30 & 50 \\
\hline \multirow{2}{*}{3} & \multirow{2}{*}{ ¿Qué cantidad compra? } & MENOS DE 1 TON & DE 1 A 5 TON & CAMION \\
\hline & & 12 & 20 & 18 \\
\hline \multirow{2}{*}{4} & \multirow{2}{*}{$\begin{array}{l}\text { ¿A qué precio compra la tonelada } \\
\text { de uva? }\end{array}$} & $5000 \mathrm{Bs}$ & $10000 \mathrm{Bs}$ & $15000 \mathrm{Bs}$ \\
\hline & & 10 & 25 & 15 \\
\hline \multirow[b]{2}{*}{5} & \multirow{2}{*}{$\begin{array}{l}\text { ¿Con que frecuencia compra } \\
\text { uva? }\end{array}$} & SEMANAL & MENSUAL & EN VENDIMIA \\
\hline & & 22 & 30 & 18 \\
\hline \multirow{2}{*}{6} & \multirow[t]{2}{*}{ ¿Compra a crédito? } & SI & \multicolumn{2}{|c|}{ No } \\
\hline & & 18 & \multicolumn{2}{|c|}{32} \\
\hline \multirow{2}{*}{7} & \multirow{2}{*}{$\begin{array}{l}\text { ¿Cree que el precio es estable y } \\
\text { adecuado? }\end{array}$} & SI & \multicolumn{2}{|c|}{ NO } \\
\hline & & 12 & \multicolumn{2}{|c|}{38} \\
\hline \multirow{2}{*}{8} & \multirow{2}{*}{$\begin{array}{l}\text { ¿La producción de uva satisface } \\
\text { la demanda actual? }\end{array}$} & SI & \multicolumn{2}{|c|}{ No } \\
\hline & & 0 & \multicolumn{2}{|c|}{50} \\
\hline \multirow[b]{2}{*}{9} & \multirow{2}{*}{$\begin{array}{l}\text { ¿Qué cantidad de toneladas está } \\
\text { dispuesto a comprar } \\
\text { semanalmente? }\end{array}$} & $10 \mathrm{tn}$ & $20 \mathrm{tn}$ & $30 \mathrm{tn}$ \\
\hline & & 5 & 15 & 30 \\
\hline
\end{tabular}


Se puede concluir que la mayor parte de los comerciantes se encuentran en los mercados de Santa Cruz, la uva que más compran es la de color verde, la cantidad de compra es de 1 a 5 toneladas y el precio que ellos adquieren es de 10.000 la tonelada cada mes. Por lo general, las compras se pagan al contado. Muchos de ellos dicen que los precios no son estables, además de que la producción actual de uva no satisface la necesidad del país y ellos están dispuestos a comprar semanalmente 30 toneladas de uva.

\section{RESULTADOS Y DISCUSIÓN}

Creación de una empresa de producción y comercialización de uva de mesa en la comunidad de Tárcana en Bolivia.

\section{Estudio de mercado}

La empresa productora de uva proveerá a las vendedoras mayoristas de los mercados de la ciudad de Santa Cruz de la Sierra del departamento de Santa Cruz, considerando que es un mercado con fuerte crecimiento de la población, industrial y comercial. Actualmente, la ciudad de Santa Cruz es un mercado que requiere de proveedores de estos frutos. Los principales proveedores de uva en esta ciudad son productores de uva de Tarija, Valle Grande, Argentina y Chile.

En los últimos años se ha visto la necesidad de importar esta fruta, ya que el $60 \%$ de la producción nacional se utiliza para la elaboración de vinos y singanis; el $35 \%$ a comercialización directa para consumo en fruta y el $5 \%$ se destinan para uvas deshidratadas (uvas pasas). La ciudad de Santa Cruz sufre de un desabastecimiento de frutas de valle, por lo cual se hace un mercado atractivo para la empresa.

\section{Oferta}

Los principales proveedores de uva a la ciudad de Santa Cruz son las regiones de Tarija, Chuquisaca, Valle Grande, también se importa producto de los países vecinos como Chile y Argentina. La producción anual de Tarija es de aproximadamente de 125.000 toneladas, por lo que este es el departamento con mayor participación en la producción de uva. Cabe resaltar que no toda la producción es destinada a la ciudad de Santa Cruz; según Wilmar Villena, productor del departamento de Tarija, afirma que solo el $20 \%$ de la producción de Tarija se destina a esta ciudad.

\section{Mix de marketing}

Producto. Uva Flame y superior.

Plaza. Mayoristas de la ciudad de mercado y supermercados de Santa Cruz.

Precio. Precios competitivos del mercado al por mayor: 10 bolivianos por kilo; en los supermercados de Santa Cruz, 10 bolivianos.

\section{Promoción}

- Dar a conocer las bondades de las uvas mediante folletos y conversaciones casuales con los clientes.

- Regalar a los clientes un reloj con una imagen de la empresa.

- Regalar calendarios con información de la producción de uva en el mundo.

\section{Canales de distribución}

Productor $\rightarrow$ Mayorista rescatista $\rightarrow$ Mayorista $\rightarrow$ Minorista $\rightarrow$ Consumidor final. 


\section{Estudio administrativo}

Naturaleza de la empresa. Empresa agrícola.

Rubro. Agropecuario.

Régimen de contribuyente. RAU (Régimen Agropecuario Unificado).

Características. Producción y comercialización de uva de la variedad Flame Seedless y Sugraone.

Tamaño. Por las características de la empresa, será una microempresa, porque contará con un trabajador, un administrador eventual y con 10 obreros eventuales para poda y 15 en cosecha.

Misión. Proveer a los comerciantes frutas producidas bajo estrictas normas de cuidado y respeto por el medioambiente, utilizando tecnología de vanguardia y cumpliendo con todos los procesos $y$ requerimientos necesarios para lograr productos de óptima calidad.

Visión. Consolidar a Viñedos La Capilla en el mercado nacional como empresa reconocida y comprometida a superar las expectativas de los clientes con los productos.

\section{Valores.}

- Compromiso con la comunidad y el medioambiente.

- Compromiso con la calidad del producto y el servicio al cliente.

- Compromiso y respeto hacia los recursos humanos.

\section{Cuadro 2. Matriz FODA}

\begin{tabular}{|l|l|}
\hline $\begin{array}{l}\text { FORTALEZAS } \\
\text {-Personal eventual y capacitado } \\
\text { disponible. }\end{array}$ & $\begin{array}{l}\text { DEBILIDADES } \\
\text { - Pinancieros }\end{array}$ \\
$\begin{array}{l}\text {-Disposición de terrenos } \\
\text { optimos }\end{array}$ & Poco interés de las personas de la \\
región por la producción de uvas
\end{tabular}

\section{Cruces estratégicos}

FO - F2: 04 02. Solicitar créditos agropecuarios a entidades financieras.

DO - D2: O2. Solicitar a una entidad financiera para que capacite e informe sobre los préstamos o financiamientos sobre el rubro.
FA - F1: A1. Adquirir equipos que evalúen los cambios climatológicos. Evaluar permanentemente a las plantas para ver si existe presencia de alguna enfermedad o plaga.

DA - D1: A1 A2. Asistir a talleres de producción de uva. 


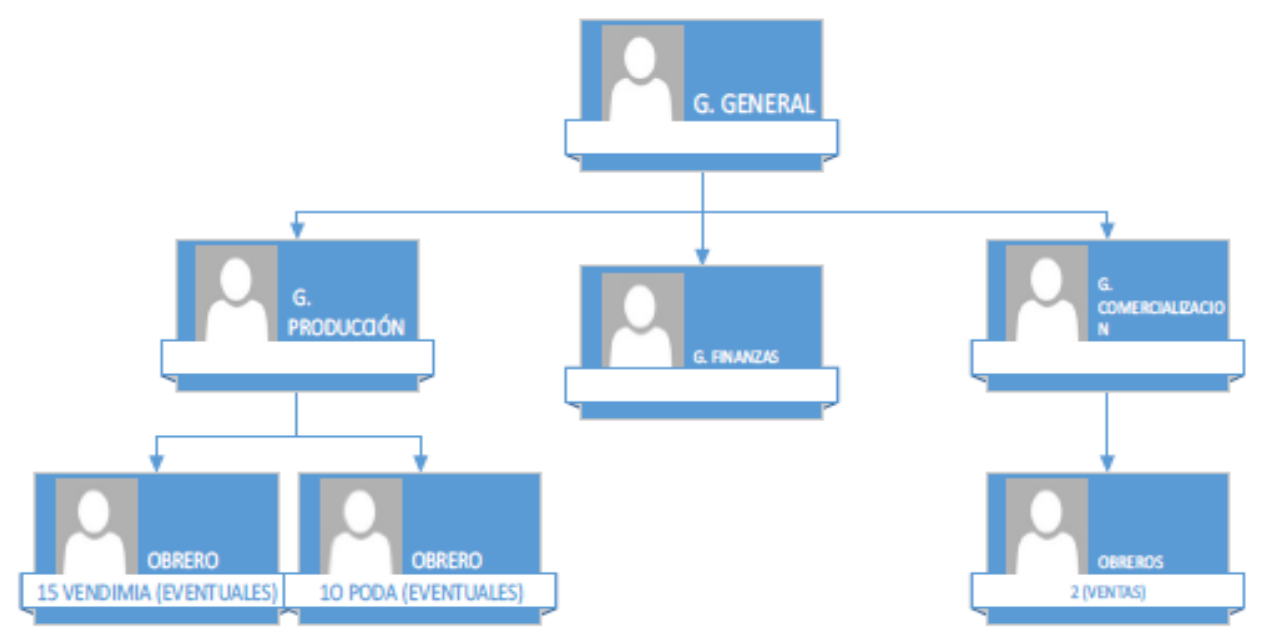

Figura 1. Fases de trabajo

\section{Proceso de producción}

\section{Etapa 1}

Preparación del terreno. Consiste en los siguientes pasos:

- Riego del terreno. Regar todo el terreno para tener un suelo blando para el cultivo.

- Arado o cultivado. Esta acción se hace con tractor. Se remueve la tierra a una profundidad de 50 centímetros.

- Abonar y fertilizar la tierra. Consiste en colocar los nutrientes y mezclar todos los abonos de manera uniforme para tener un suelo fértil. Esta actividad es periódica durante toda la vida de los viñedos.

- Oxigenación del suelo. Consiste dejar en reposo de 20 a 30 días para que el subsuelo se oxigene.

- Excavación de los agujeros. Hacer los agujeros y dejarlos listos para plantar los plantines.

\section{Etapa 2}

Establecimiento del sistema de conducción (parrón español). Para la variedad de uva que se decidió producir, se seleccionó el sistema de parrón español, que consiste en:

- Colocar postes esquineros perimetrales y postes tutores.

- Colocar una malla de alambre que unen los postes tutores perimetrales y esquineros.

\section{Etapa 3}

Establecimiento del sistema de malla antigranizo. Se extiende a través de las hileras una red que proteja las plantas de las granizadas, para evitar que estas sean dañadas.

\section{Etapa 4}

Plantar los plantines. Consiste en plantarlos, abonarlos y hacer el primer riego. Se riega de manera regular desde la primavera hasta el mes de abril o un mes después de la cosecha. Después de esta actividad, se suspenden los riegos para que la planta duerma o descanse en el invierno. 


\section{Etapa 5}

Crecimiento o desarrollo. Consiste en guiar la planta mediante:

- Poda de formación. Se efectúa después de 40 o 60 días de la siembra. Si se ha sembrado la planta ya formada en fundas, se elige el brote más vigoroso y mejor ubicado para formar el tronco. Se deschupona eliminando las hojas que apareen en las axilas de las hojas principales los brotes laterales. Debe amarrarse al tutor o poste cada $20 \mathrm{~cm}$. Cuando el brote principal sobrepasa 30 $\mathrm{cm}$, la malla se despunta a $10 \mathrm{~cm}$ por debajo de la malla, para inducir el desarrollo de los brotes laterales. Por lo general, se escogen 4 brotes opuestos en forma de cruz, estos se van despuntando cada $50 \mathrm{~cm}$, para que a su vez se desarrollen brotes laterales $\mathrm{y}$ futuros cargadores (ramas productivas de frutos) el largo de los brazos varía de 1 a 1,5 metros dependiendo de la distancia de la siembra. Debe evitarse la formación de dos troncos por planta.

- Poda de producción. La poda de producción se repite cada ciclo vegetativo de la planta. Se escoge una yema bien formada y se corta el sarmiento formado en el ciclo vegetativo anterior a $1 \mathrm{~cm}$ por encima de ella. La parte del sarmiento que se deja adherida a la planta es el cargador y su longitud depende principalmente del hábito de fructificación de la variedad o ya sea por la posición de las yemas fructíferas y el tamaño de los racimos. Se recomienda que se pode de manera que los cargadores tengan de 4 a 6 yemas. Consiste en establecer un modelo a la planta para que esta vaya desarrollándose y pueda producir. Esta etapa se lleva a cabo desde el segundo año al tercero, dependiendo del tamaño que haya alcanzado la planta.

- Poda en verde. Esta tiene como objetivo preparar la planta para que tenga una producción uniforme de los frutos. Consiste en el desbrote o eliminación de los brotes inútiles en los troncos y brazos y el despunte o corte de los extremos de los sarmientos a una longitud de 10 a 20 $\mathrm{cm}$. Otro propósito que tiene es que los nutrientes de la planta sean dirigidos a los frutos y no así a los tallos y hojas. Se poda desde que la planta empieza a producir hasta que esta deja de hacerlo. Los riegos en esta etapa se hacen de manera regular.

\section{Etapa 6}

Producción. Esta etapa empieza en la primavera, en el mes de septiembre, con la floración de las plantas.

\section{Etapa 7}

Cosecha. Se recortan los racimos maduros o pintados en cajas plásticas de aproximadamente de 8 a $10 \mathrm{~kg}$. Luego son trasladados hasta el galpón o vehículo que transportará el producto hasta el mercado final.

\section{Etapa 8}

Comercialización. Se traslada el producto hasta el punto de intercambio, que puede ser el mismo lugar donde se produce, mercados de la ciudad de Santa Cruz y los supermercados.

\section{Estudio financiero}

El objetivo del estudio financiero es determinar la inversión, costos de producción, comercialización, los ingresos para determinar su factibilidad y viabilidad del proyecto. 
Tabla 1. Inversiones (expresado en bolivianos)

\begin{tabular}{|c|c|c|c|c|}
\hline $\mathrm{N}^{\circ}$ & DETALLE & \multicolumn{2}{|c|}{ FUENTE } & TOTAL Bs. \\
\hline \multirow{12}{*}{1} & & INTERNA & EXTERNA & \\
\hline & INVERSIÓN FIJA & & & \\
\hline & Terreno & 300,785 & 0 & 300,785 \\
\hline & Maquinaria y equipo & 0 & 314,232 & 314,232 \\
\hline & Construcciones civiles & 0 & 33,100 & 33,100 \\
\hline & Muebles y enseres & 0 & 9,350 & 9,350 \\
\hline & Vehículo & 0 & 276,312 & 276,312 \\
\hline & Vinedo & 0 & 9,350 & 9,350 \\
\hline & Equipo de computación & 0 & 19,450 & 19,450 \\
\hline & Herramientas & 0 & 3,580 & 3,580 \\
\hline & Vides & 86,700 & 0 & 86,700 \\
\hline & $\underline{\text { TOTAL }}$ & $\underline{\underline{387.485}}$ & $\underline{665,374}$ & $\underline{1,052,859}$ \\
\hline \multirow{3}{*}{2} & INVERSIÓN DIFERIDA & & & \\
\hline & Gastos de organización & 1,202 & 0 & 1,202 \\
\hline & TOTAL & $\underline{\underline{1,202}}$ & $\underline{0}$ & $\underline{1,202}$ \\
\hline \multirow{13}{*}{3} & CAPITAL DE TRABAJO & & & \\
\hline & Gastos de puesta en marcha & 9,200 & 0 & 9,200 \\
\hline & Gestión de compras & 1,000 & 0 & 1,000 \\
\hline & Insumos & 26,150 & 0 & 26,150 \\
\hline & Seguridad industrial & 16,750 & 0 & 16,750 \\
\hline & Lubricantes y combustibles & 49,600 & 0 & 49,600 \\
\hline & Material de escritorio & 637 & 0 & 637 \\
\hline & Material de limpieza & 190 & 0 & 190 \\
\hline & Utensilios de cocina & 1,140 & 0 & 1,140 \\
\hline & Gastos operativos & 180,000 & 0 & 180,000 \\
\hline & Servicios básicos & 900 & 0 & 900 \\
\hline & TOTAL & 285,567 & 0 & 285,567 \\
\hline & INVERSIÓN TOTAL & $\underline{674,254}$ & $\underline{665,374}$ & $\underline{1,339,628}$ \\
\hline
\end{tabular}

\section{Discusión}

El estudio de mercado de un proyecto es uno de los análisis más importantes y complejos que debe llevar a cabo el investigador. Más que centrar la investigación en el consumidor y la cantidad del producto que demandará, tiene que estudiar los mercados, proveedores, competidores y distribuidores, e incluso cuando se requiera, las condiciones del mercado externo. El estudio, más que describir y proyectar los mercados destacados para el proyecto, debe ser una base sólida para realizar una investigación completa; debe arrojar datos básicos para las demás partes de la investigación (Hernández y Hernández, 2008).
Según Paredes (2009), el mercado es una institución a través de la cual operan la demanda y oferta, fuerzas determinantes de los precios. Los objetivos que persigue el estudio de mercado son encontrar mercados rentables, elegir productos que se puedan vender, identificar a los consumidores (personas y/o empresas) del bien o servicio ofrecido.

En economía, un consumidor es una persona u organización que demanda bienes o servicios proporcionados por el productor o el proveedor de bienes o servicios. Es decir, es un agente económico con una serie de necesidades (Ovalle, 2000).

Se define la demanda de un bien o servicio como las diferentes cantidades del 
producto que los consumidores estarán dispuestos a adquirir en el mercado a los diferentes precios alternativos posibles, siempre y cuando los otros factores permanezcan constantes (Paredes, 2009).

La oferta hace referencia a la cantidad de unidades de un producto que las empresas de bienes o servicios estarían dispuestas a intercambiar a un precio determinado; para una demanda dada habrá una oferta determinado; entonces, para establecer ofertas de valor, es importante entender los mercados y sus necesidades (Díaz, 2011).

El precio es una variable económica de mucha importancia en el estudio de mercado, por cuanto es el parámetro que muestra la cantidad monetaria a la cual los productores están dispuestos a vender y los demandantes a comprar un bien o servicio, $y$ la comercialización es el mecanismo mediante la cual un bien o servicio llega al consumidor, es decir, en esta parte se determina la forma de transferencia del producto ofrecido por el proyecto al demandante final (Ramallo, 2005).

Con base en los resultados obtenidos del estudio de mercado - y concretamente de la demanda insatisfecha de un bien o servicio en un área de influencia del proyecto-, se determina el tamaño de la unidad de producción, es decir, la capacidad real y efectiva de producción. En tal sentido, existen tecnologías llamadas modulares que permiten ir adaptando la capacidad instalada a la demanda del mercado. Otras tienen tamaños mínimos de capacidad predeterminados $\mathrm{y}$, en algunos casos, se hace necesario instalar capacidades mayores a las requeridas. Esto se hace posible cuando los beneficios son superiores a los costos aún bajo estas condiciones. Por ello, señalamos que la tecnología influye en la determinación del tamaño. La disponibilidad financiera debe ser tomada muy en cuenta en la determinación del tamaño. $O$ en el desarrollo o ampliaciones futuras de la empresa. La capacidad financiera de las inversiones fija un límite máximo de inversión, del cual se desprende el tamaño del proyecto.

Por su parte, Bacca (2010) expresa que para favorecer la decisión correspondiente al donde, resulta de gran ayuda algunos factores que facilitan dicha elección. Su ordenamiento no reviste la importancia de ellos, por cuanto para cada proyecto los factores pueden cambiar de importancia, a saber: clima, servicios, materia prima, mano de obra, terrenos y mercados.

Para Funes (2012), se entiende por empresa al organismo social integrado por elemento humanos, técnicos y materiales cuyo objetivo natural y principal es la obtención de utilidades, o bien la prestación de servicios a la comunidad, coordinados por un administrador que toma decisiones de forma oportuna para la consecución de los objetivos para los que fueron creadas.

Es importante ahora señalar que la inversión fija -denominada también inversión en activos tangibles - comprende todos aquellos bienes que adquieren durante la etapa de instalación del proyecto. Estas inversiones son bienes materiales que se tocan y se ven, intervienen en el proceso de transformación de la materia prima o sirven de apoyo en las actividades productivas del proyecto. Normalmente, la inversión fija suele ser la más elevada de todos los componentes de la inversión total; en su valoración se incluyen todos los gastos de constitución de las obras físicas, de adquisición, transporte y montaje de equipos y maquinarias (Paredes, 2009). 
Este autor considera como capital de trabajo aquellos recursos que requiere el proyecto para atender las operaciones de producción y comercialización de bienes o servicios, y complementa el monto de dinero que se precisa para dar inicio al ciclo productivo del proyecto en su fase de funcionamiento. En otras palabras, es el capital adicional con el que se debe contar para que comience a funcionar el proyecto, esto es, financiar la producción antes de percibir ingresos.

\section{CONCLUSIONES}

El estudio de mercado demostró que la producción y comercialización es favorable para la comercialización de uva. Además, que se pueden hacer uso de las nuevas tecnologías que están disponibles en el mercado como son los sistemas de conducción riegos a gateo. También se demostró que existen disponibilidad y acceso rápido a los proveedores de insumos, así como de las maquinarias para ser utilizados en la ejecución del proyecto.

El estudio administrativo propuso un modelo de organigrama eficiente y eficaz que permitirá emplear al personal adecuado en cada puesto de trabajo para que este se pueda desenvolver adecuadamente según a las funciones que le corresponden. Como así también se propuso una misión y la visión para que el personal de nuestra empresa sepa quiénes somos que hacemos y hacia donde apuntamos en el futuro, además de los valores por el cual estará sujeta nuestra empresa en su entorno.

El estudio técnico reveló que existen las condiciones favorables para la puesta en marcha del proyecto, la experiencia de los integrantes de esa comunidad. También se investigó que los problemas que existen actualmente en el lugar pueden ser controlados si se hacen las actividades correspondientes en su debido momento. En este sentido el proyecto elaborado tiene todas las condiciones de prevención sobre los problemas encontrados en dicho proyecto.

Los resultados obtenidos del estudio financiero muestran que es factible la creación de una empresa productora de uva. Arrojando datos atractivos para cualquier inversionista según el análisis realizado.

Los que desean ejecutar dicho proyecto deberán tomar en cuenta las siguientes recomendaciones:

- Seguir el proceso desarrollado en el proyecto para tener un mejor resultado en la producción.

- Actualizar los datos sobre la fecha ejecutable, por el hecho de que los datos fueron extraídos cuando se elaboró el proyecto.

- El proyecto está dirigido para la comunidad de Tárcana. Si se quiere ejecutar en otro lugar, tomar en cuenta las variables del lugar donde se ejecutará el proyecto.

- Para aprovechar los mejores precios del mercado, poner uvas que maduren de diciembre a enero (tempraneras).

- Construir una cámara de refrigeración para guardar la uva y así poder venderla en época que no haya producción.

- Utilizar un sistema de recepción de pedido y de despacho para agilizar las actividades de comercialización.

- Llevar un registro del proceso para tener un panorama general acerca de las acciones a llevar a cabo en posteriores años o ciclos de producción.

- Analizar el avance del proyecto para tomar medidas adecuadas y oportunas en las plantas para evitar cualquier 
inconveniente imprevisto que no se haya tomado en cuenta en dicho proyecto.

\section{REFERENCIAS}

Bacca, G. (2010). Evaluación de proyectos. México: McGraw-Hill/Interamericana Editores.

Castillo, M. (2004). Guía para la formulación de proyectos de investigación. Colombia: Cooperativa Editorial Magisterio

Colciencias (2010). Servicio Permanente de Indexación de Revistas de Ciencia, Tecnología e Innovación Colombianas. Colombia: Recuperado de: http://www.colciencias.gov.co/sites/defa ult/files/upload/paginas/M304PR02G01 guiaserviciopermanente-indexacion.pdf
Díaz, Á. (2011). Dirección de proyectos. Experiencia, arte y excelencia. Colombia: Alfa Omega Grupo Editor

Funes, J. (2012). El ABC de la contabilidad (8a ed.). Bolivia: Sabiduría y Cultura.

Hernández, A. y Hernández, S. (2014). El Alto del departamento de La Paz, gestión 2014. Universidad Adventista de Bolivia

Ovalle J. (2000). Derechos del consumidor. Cámara de Diputados, LVIII legislatura. Universidad Nacional Autónoma de México

Paredes, R. (2009). Elementos de elaboración $y$ evaluación de proyectos (3a ed.). Bolivia: Imp. Publicidad San Jinés

Ramallo, A. (2005). Preparación, evaluación y administración de proyectos de inversión, enfoque de sistemas. Bolivia: Latina Editores 